\title{
Rol del virus papiloma humano en cabeza y cuello
}

\author{
Role of human papillomavirus in head and neck
}

\author{
Carolina Oliva G. ${ }^{1}$, Francisco Pérez D. ${ }^{2}$, Ricardo Zamorano S. ${ }^{1,3}$, Paul Boettiger B. ${ }^{1,4}$
}

\begin{abstract}
Resumen
El virus papiloma humano (VPH) es un virus de ADN circular doble hebra con alrededor de 200 genotipos. Este virus se relaciona a distintas lesiones tumorales que afectan cabeza y cuello, incluyendo lesiones malignas escamosas causadas por genotipos de alto riesgo como VPH-16 y VPH-18. Esta revisión tiene por objetivo determinar el rol del VPH en distintas patologías, haciendo distinción entre lesiones benignas y malignas, y con particular énfasis en aquellas en que se ha demostrado una asociación causal con el virus, tales como papilomatosis laríngea y carcinoma escamoso orofaríngeo. Además, se analizarán los mecanismos moleculares de daño, métodos de detección y de prevención como la vacunación contra el virus. Es necesario conocer la relevancia del VPH en nuestra especialidad, ya que su determinación puede tener implicancias en cuanto al manejo y pronóstico de los pacientes.
\end{abstract}

Palabras clave: virus papiloma humano, papilomatosis laríngea, carcinoma orofaringe, carcinoma rinosinusal, carcinoma laringe.

\begin{abstract}
Human papillomavirus (HPV) is a double stranded circular DNA virus with around 200 genotypes. This virus is related to different tumor lesions that affect the head and neck, including malignant squamous lesions caused by high-risk genotypes such as HPV-16 and $H P V-18$. The objective of this review is to determine the role of HPV in different pathologies, distinguishing between benign and malignant lesions, and with particular emphasis on those in which a causal association with the virus has been demonstrated, such as laryngeal papillomatosis and oropharyngeal squamous carcinoma. In addition, molecular damage mechanisms, detection and prevention methods such as vaccination against the virus will be analyzed. It is necessary to know the relevance of HPV in our specialty, since its determination may have implications in terms of patient management and prognosis. Keywords: Human papillomavirus, laryngeal papillomatosis, oropharyngeal carcinoma, rhinosinusal carcinoma, laryngeal carcinoma.
\end{abstract}

${ }^{1}$ Servicio de Otorrinolaringología, Hospital Clínico Universidad de Chile. Santiago, Chile. ${ }^{2}$ Facultad de Medicina, Universidad de Chile. Santiago, Chile.

${ }^{3}$ Servicio de Otorrinolaringología, Clínica Santa María. Santiago, Chile. ${ }^{4}$ Servicio de Otorrinolaringología, Clínica Indisa. Santiago, Chile.

Los autores declaran no tener conflictos de interés.

Recibido el 28 de abril de 2020 Aceptado el 1 de diciembre de 2020

Correspondencia: Carolina Oliva G. Santos Dumont 999, Independencia Departamento Otorrinolaringología Hospital Clínico de la Universidad de Chile Santiago, Chile. Email: carolinaolivaguerrero@ gmail.com

\section{Introducción}

El virus papiloma humano (VPH), miembro de la familia Papillomaviridae, es un virus pequeño con simetría icosaédrica, cuyo genoma posee un ADN circular doble hebra. La familia VPH comprende alrededor de 200 genotipos, la mayoría de los cuales causan lesiones benignas autolimitadas, sin embargo, un pequeño número clasificado como de alto riesgo (VPH-AR) son agentes causales de distintas malignidades, que incluyen cáncer cervicouterino, anogenital y orofaringe $e^{1}$. Los genotipos más frecuentes asociados a cáncer son VPH-16 y VPH-18, otros subtipos de alto riesgo son 31 , 33, 35, 39, 45, 51, 52, 56, 58 y $59^{2}$.

El ciclo de vida del VPH está ligado a la diferenciación del tejido epitelial del huésped. El virus infecta a las células del epitelio basal a través de heridas localizadas o microabrasiones ${ }^{3}$. Después de la infección, el genoma viral se mantiene en el núcleo de las células basales de forma episomal (extracromosomal) expresando los genes tempranos E1-E8, cuando estas células se dividen algunas se mueven hacia la capa suprabasal del epitelio y comienzan su 
proceso de diferenciación. Estas células al ser diferenciadas normalmente deberían estar fuera del ciclo celular, sin embargo, el VPH activa la maquinaria de síntesis de DNA celular mediante la expresión de E6 y E7 y permite la amplificación del genoma viral ${ }^{4}$. Las proteínas de fase tardía L1 y L2 encapsidan el DNA viral sintetizado y los viriones maduros son liberados desde la capa más superficial del epitelio (Figura 1)5. Uno de los signos distintivos de los tumores inducidos por VPH es la expresión continua de las oncoproteínas virales E6 y E7, generalmente causada por la integración del virus en el genoma del hospedador ${ }^{1}$.

\section{Condiciones no asociadas a cáncer}

\section{Lesiones benignas de cavidad oral y orofaringe}

La frecuencia de la infección por VPH en mucosa sana es del 3\% a 5\% en adolescentes, aumentando a un $5 \%$ a $10 \%$ en adultos ${ }^{6}$. Su transmisión puede ser por distintas vías: sexual y no sexual, aunque estudios indican que la prevalencia en adultos aumenta conforme al número de parejas sexuales sobre todo en hombres, pacientes VIH positivos y consumidores de tabaco ${ }^{7}$. Las lesiones benignas son:

\section{a) Papiloma escamoso}

Causado en su mayoría por VPH-6 y VPH-11 a cualquier edad, afecta a 1 en 250 adultos. Se caracteriza por ser una lesión exofítica verrucosa y corresponde a la lesión tumoral benigna más frecuente de la cavidad oral, afectando con mayor frecuencia paladar blando, superficie ventral de la lengua, frenillo lingual, y mucosa labial. Pese a su relativa frecuencia, la literatura acerca del manejo es escasa, sin embargo, se recomienda la resección de la lesión como tratamiento. Además, se ha visto la presencia de displasia en algunas de estas lesiones, lo que apoya un tratamiento quirúrgico. La recurrencia de estas lesiones es inusual y se cree que es causada por remoción incompleta del epitelio infectado en la base de la lesión. La reinfección continua o la transición de una lesión subclínica a clínica puede causar la aparición de nuevas lesiones ${ }^{8}$.

\section{b) Verruga vulgaris}

Lesión contagiosa causada por VPH-2 y VPH-4, afecta la piel y regiones queratinizadas de la cavidad oral como labios, paladar y encías. Pueden aparecer a cualquier edad, pero principalmente durante la niñez. El diagnóstico diferencial con papiloma escamoso no tiene importancia desde el punto de vista clínico y el tratamiento es el mismo ${ }^{8}$.

\section{c) Hiperplasia epitelial focal}

También llamada enfermedad de Heck, está asociada a VPH-13 y VPH-32 en un 7\% a 36\%. Afecta principalmente a niños y a pacientes $\mathrm{VIH}$ positivos. Se caracteriza por múltiples lesiones nodulares localizadas en la mucosa de los labios y lengua. Las lesiones desaparecen sin tratamiento después de un largo tiempo, su recurrencia es poco frecuente ${ }^{9}$.

Figura 1. Ciclo de vida del VPH en el epitelio.

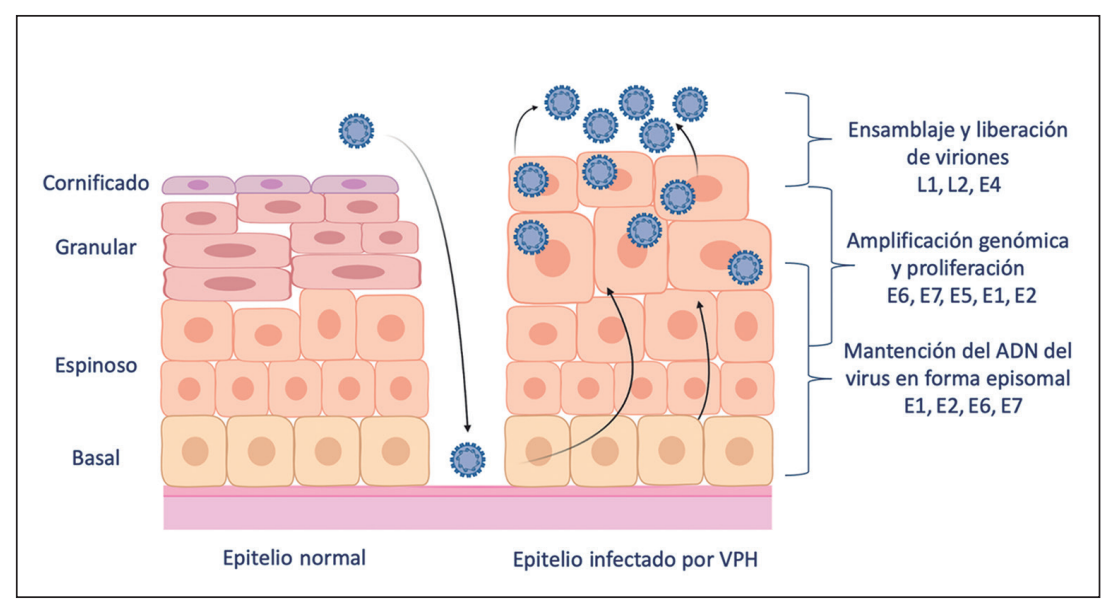




\section{d) Condiloma acuminado}

Provocado por VPH-6, VPH-11 y VPH-2, en la cavidad oral afecta regiones asociadas a contacto orogenital como la mucosa labial, paladar blando y frenillo lingual, también se encuentra en laringe y tráquea. Es más frecuente en personas con condilomas genitales. Son difíciles de tratar y se debe realizar resección quirúrgica de todas las lesiones para reducir la probabilidad de recurrencia ${ }^{6,8}$.

\section{Papilomatosis laríngea}

La papilomatosis laríngea o papilomatosis respiratoria recurrente (PRR) es una neoplasia benigna de la vía respiratoria, principalmente de la laringe, cuya causa está fuertemente asociada a la infección por $\mathrm{VPH}^{10}$. La PRR se clasifica respecto a su edad de presentación en papilomatosis respiratoria recurrente de "inicio juvenil” (PRR-IJ) y la forma adulta (PRRIA). En cuanto a su etiología, los genotipos de bajo riesgo 6 y 11 de VPH son los principales implicados ${ }^{11}$, sin embargo, también han sido asociados los genotipos $16,18,31$ y $33^{12}$. La forma de contagio aún no está esclarecida, pero se postula que en la PRR-IJ, el virus se transmitiría por contacto de secreciones durante el parto en mujeres con infección activa de VPH. Por otro lado, en adultos, se sugiere que puede ser producto de una reactivación de la infección adquirida a temprana edad o haber sido originada en un contacto por medio de sexo oral $^{13}$. Los pacientes infectados por VPH-11 presentan un cuadro más agresivo en comparación con los infectados con VPH-6, sin embargo, la recomendación de la genotificación no está clara aún ${ }^{14}$. La transformación maligna es infrecuente y se ha asociado en limitados casos a VPH de bajo riesgo ${ }^{15}$, sin embargo, se sugiere que la transformación a carcinoma laríngeo está asociada principalmente a factores de riesgo como tabaquismo, consumo de alcohol, inmunosupresión y radiación ${ }^{11}$. Por otra parte, varios estudios han sugerido la co-infección con genotipos 16 y 18 de VPH como un importante factor de transformación maligna $^{16}$.

La vacunación contra VPH es una alternativa para prevenir las consecuencias del virus, sin embargo, es poca la información acerca de la prevención contra $\mathrm{PRR}^{10}$. Un reporte del seguimiento a 5 años del programa de va- cunación de Australia informa que la tasa de incidencia de PRR ha disminuido en un factor de 0,614 por año posterior a la implementación de la vacuna contra $\mathrm{VPH}^{17}$. Esta es la primera aproximación del uso de la vacuna para la erradicación de PRR. A su vez, un metaanálisis de 22.474 pacientes reportó una reducción de la recurrencia de PRR y mayor intervalo de reintervención, luego de la aplicación de la vacuna cuadrivalente contra el virus de forma adyuvante a la cirugía, en casos de patología clínicamente activa ${ }^{18}$. Posteriormente se retomará este tema en el ítem de prevención.

\section{Papiloma invertido}

El papiloma invertido es un tumor benigno de la cavidad nasal y senos paranasales, frecuentemente localizado en la pared lateral de meato medio. Se presenta como una masa unilateral, con una alta tendencia a la recurrencia y transformación maligna ${ }^{19}$. Desde el punto de vista fisiopatológico algunos autores han propuesto que el VPH tendría un rol en la etiología del papiloma invertido y progresión a carcinoma escamoso, particularmente a través del aumento de expresión de EGFR ${ }^{20-22}$. Sin embargo, estudios retrospectivos con muestras tumorales describen una positividad del 5,1\%$13 \%$ para la presencia de VPH en general, 5\%-6\% para VPH-AR; baja correlación entre la inmunohistoquímica para p16 y la presencia de VPH en estos tumores, además de no existir relación entre el virus y la recurrencia o desarrollo de carcinoma escamoso ${ }^{19,23,24}$. En conclusión, no es posible establecer al VPH como un factor etiológico ni de progresión en papiloma invertido.

\section{Cáncer y VPH}

La prevalencia del VPH es altamente variable y dependiente del subsitio anatómico y la región. Considerando todos los carcinomas de cabeza y cuello, aproximadamente el 25\% constituyen tumores VPH positivos ${ }^{25}$, con mayor prevalencia en el carcinoma orofaríngeo con un $46 \%$, seguido de $24 \%$ en cavidad oral y $22 \%$ en cáncer laríngeo ${ }^{26}$. En mujeres, la mayoría de los tumores asociados a VPH corresponden al área ginecológica, sin embargo, en hombres, los carcinomas de cabeza 
y cuello VPH-positivos corresponde al 50\% de los cánceres asociados a VPH en este género, siendo diagnosticado casi 3 veces más en países desarrollados en comparación a aquellos menos desarrollados ${ }^{27}$. Daremos un mayor énfasis al carcinoma orofaríngeo en esta revisión, ya que se ha demostrado un rol oncogénico del $\mathrm{VPH}$ en esta entidad.

\section{Detección de VPH en muestras histopatológicas}

Existen distintos métodos de análisis para $\mathrm{VPH}$, el más utilizado en la clínica debido a su facilidad, costo-efectividad y bajo material requerido es la inmunohistoquímica para la detección de la proteína p16, marcador sustituto de la presencia del virus. Es particularmente útil en carcinoma orofaríngeo con una sensibilidad del $94 \%$ y especificidad de $83 \%{ }^{28}$, en ellos el 1\%-7\% de estos tumores con tinción negativa para p16 son VPH positivos, sin embargo, en otros tumores este porcentaje se eleva considerablemente perdiendo fidelidad para el virus. En muestras tumorales también puede emplearse la reacción de polimerasa en cadena (PCR por sus siglas en inglés) de DNA viral extraído, lo que permite también realizar análisis de él o los genotipos implicados. Otros métodos propuestos que indicarían actividad biológica en el proceso de carcinogénesis son el análisis de expresión de transcritos E6/E7 (indicador de transcripción viral oncogénica activa) mediante PCR transcriptasa reversa y la hibridación in situ que demostraría la integración del virus al genoma del huésped, paso necesario para la transformación oncogénica celular; sin embargo, estos métodos se caracterizan por ser más dificultosos técnicamente, de mayor costo, no estar implementados en todos los laboratorios y tener menor sensibilidad. Considerando estos antecedentes, en biopsias obtenidas de los subsitios de la orofaringe bastaría la inmunohistoquímica para p16, para catalogar como VPH positivo. En caso de metástasis cervical de primario desconocido, el Colegio Americano de $\mathrm{Pa}$ tólogos señala que las muestras obtenidas de los niveles II y III, con tinción p16 positiva asociada a morfología no queratinizante indican la presencia de VPH, no obstante, si la morfología es queratinizante es necesario complementar con métodos moleculares adicionales ${ }^{29}$. Debido a la extensión del artículo sugerimos que, en caso de que el lector quiera profundizar en la interpretación de p16 en muestras obtenidas a través de punción con aguja fina, tumores en otros subsitios no orofaríngeos y la complementación con otras técnicas, acuda al artículo de Paver y cols. ${ }^{29}$ para su complementación.

\section{Carcinogénesis}

Uno de los signos distintivos de tumores inducidos por VPH es la expresión continua de las oncoproteínas virales E6 y E7, causada por la integración del virus en el genoma del hospedador. La oncoproteína E6 recluta a la proteína asociada a E6, una ubiquitina ligasa que gatilla la degradación de p53, desregulando los puntos de control G1/S y G2/M típicamente inducidos por daño en el DNA. La oncoproteína E6 induce, además, la expresión y actividad de hTERT que es la subunidad catalítica de la telomerasa favoreciendo así, la inmortalización celular. Por otra parte, E7 promueve la transformación celular mediante la degradación de la proteína retinoblastoma $(\mathrm{Rb})$, liberando el factor transcripcional E2F y subsecuente estimulación de la fase $\mathrm{S}$ del ciclo celular, promoviendo además la sobreexpresión de la proteína $\mathrm{p} 16$, otra característica presente sobre todo en carcinoma orofaríngeo, utilizado como marcador sustituto del $\mathrm{VPH}^{30,31}$. Si bien, estos son los principales mecanismos de transformación y progresión maligna, existen múltiples mecanismos adicionales involucrados $^{1}$. En la Figura 2 se esquematiza el rol de las proteínas mencionadas.

\section{Cáncer de orofaringe}

\section{a) Epidemiología}

Se ha observado un incremento de incidencia del cáncer de cabeza y cuello VPH positivo en las últimas décadas, concomitantemente con una disminución en el consumo de tabaco, en particular en cánceres de orofaringe VPH positivos en hombres jóvenes en el norte de Europa y Estados Unidos ${ }^{32,33}$. Se presume que este aumento está determinado por conductas sexuales de riesgo, tales como múltiples parejas sexuales y contacto sexual oral ${ }^{34,35}$. A diferencia del carcinoma cérvico-uterino donde HPV-16 y HPV-18 son los causantes 
del 70\% de los cánceres, más del 90\% de los carcinomas orofaríngeos son causados por VPH- $16^{36}$.

\section{b) Sitio anatómico}

La orofaringe está compuesta por las amígdalas palatinas, base de la lengua, paladar blando, úvula, amígdala lingual, las paredes anteriores, laterales y posteriores de la faringe ${ }^{37}$, aunque el virus afecta con mayor frecuencia las dos primeras mencionadas. El virus presenta tropismo por las células del epitelio reticular presente en las criptas palatinas, las cuales son susceptibles a la transformación y podrían ser un análogo a la zona de transición cérvico-uterina ${ }^{3}$. Un estudio reciente de 23.297 pacientes demuestra que el subsitio afectado es un factor pronóstico independiente, siendo los carcinomas no tonsilares de peor pronóstico de supervivencia ${ }^{38}$.

\section{c) Etapificación}

El carcinoma orofaríngeo VPH positivo posee un perfil epidemiológico, molecular y clínico distinto a aquellos carcinomas asociados a consumo de tabaco y alcohol. Ang y cols. ${ }^{39}$ clasificaron a los pacientes en categorías de riesgo de muerte, siendo el estatus VPH positivo de bajo riesgo en caso de índice paquete año (IPA) $<10$, o bien, IPA $>10$ pero $\mathrm{N}$ no mayor a $\mathrm{N} 2 \mathrm{a}$; $y$ pacientes de riesgo intermedio en aquellos con estatus VPH positivo, IPA > 10 y N2b-N3. El estudio multicéntrico ICON-S que incluyó a 1907 pacientes con carcinoma orofaríngeo VPH positivo propuso una etapificación de acuerdo con el pronóstico $^{40}$ que, posteriormente fue acogida por la $8^{\text {va }}$ edición del sistema de etapificación de la American Joint Committe on Cancer, separa en dos entidades distintas el carcinoma orofaríngeo p16 positivo y negativo (Tabla 1) ${ }^{41}$. Este cambio ha resultado en que la mayoría de los pacientes clasificados como etapa III-IV ahora correspondan a etapa I-II, reflejando el alto grado de supervivencia global y libre de enfermedad a los dos años postratamiento ${ }^{42}$.

\section{d) Tratamiento}

A pesar de avances significativos en técnicas quirúrgicas como la cirugía robótica, radioterapia y fármacos, el aumento del $\mathrm{VPH}$ durante las últimas dos décadas ha sido el

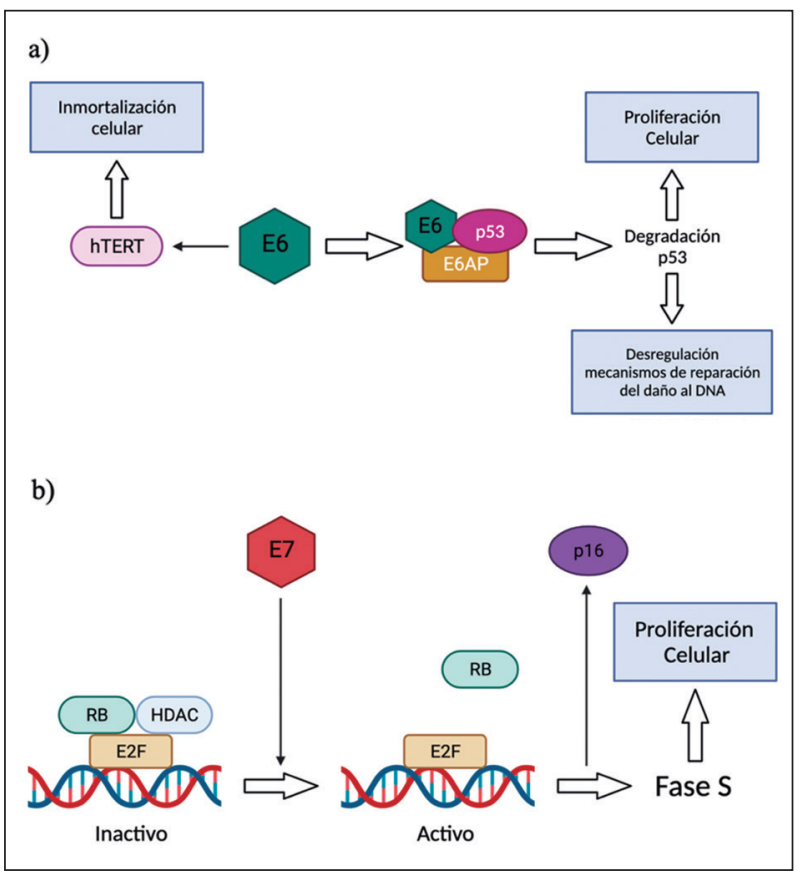

Figura 2. En a) y en b) se muestran los mecanismos simplificados en el proceso de carcinogénesis de las oncoproteínas E6 y E7 respectivamente.

principal agente de mejoría en la supervivencia. Dado este buen pronóstico en el ámbito de investigación existen esfuerzos de de-escalamiento de la intensidad del tratamiento para disminuir la toxicidad aguda y crónica ${ }^{42}$, lo que es particularmente importante considerando que estos pacientes son más jóvenes que aquellos VPH negativos. Estudios han utilizado distintas estrategias, tales como reducción de dosis de radioterapia, reducción de volumen irradiado, quimioselección para disminuir la subsecuente radioterapia, evitar la resección, cirugía transoral, omisión de terapia adyuvante, entre otras ${ }^{43}$. A pesar de estos esfuerzos, no existen guías para la elección de terapia apropiada en pacientes con carcinoma orofaríngeo $\mathrm{VPH}$ positivo, y debe aplicarse tratamiento para todos estos tumores de acuerdo a su etapificación independiente de su estatus viral, situación que podría cambiar en un futuro próximo con mayor investigación y abordaje multidisciplinario ${ }^{43}$. Las distintas investigaciones en relación con el tratamiento en carcinoma orofaríngeo positivo escapan de los objetivos de este artículo. 


\begin{tabular}{|c|c|c|c|}
\hline \multicolumn{2}{|c|}{$\begin{array}{l}\text { Carcinoma de orofaringe no asociado a VPH } \\
\text { (p16 negativo) }\end{array}$} & \multicolumn{2}{|c|}{$\begin{array}{l}\text { Carcinoma de orofaringe asociado a VPH } \\
\text { (p16 positivo) }\end{array}$} \\
\hline Estadio 0 & Tis, NO, MO & \multirow[t]{2}{*}{ Estadio I } & \multirow[t]{2}{*}{ TO a T2, NO o N1, MO } \\
\hline Estadio I & T1, No, Mo & & \\
\hline Estadio II & $\mathrm{T} 2, \mathrm{NO}, \mathrm{MO}$ & Estadio II & $\begin{array}{l}\mathrm{T} 0 \text { a T2, N2, M0 } \\
\text { T3, NO o N2, M0 }\end{array}$ \\
\hline Estadio III & $\begin{array}{c}\text { T3, N0, M0 } \\
\text { T1 a T3, N1, M0 }\end{array}$ & Estadio III & $\begin{array}{c}\text { Cualquier } \mathrm{T}, \mathrm{N} 3, \mathrm{M} 0 \\
\text { Cualquier } \mathrm{T} \text {, cualquier } \mathrm{N} \text {, } \\
\mathrm{M} 0\end{array}$ \\
\hline Estadio IVA & $\begin{array}{c}\text { T4a, N0 o N1, M0 } \\
\text { T1 a T4a, N2, M0 } \\
\text { IV B }\end{array}$ & \multirow[t]{3}{*}{ Estadio IV } & \multirow[t]{3}{*}{$\begin{array}{c}\text { Cualquier T, cualquier } \mathrm{N}, \\
\mathrm{M} 1\end{array}$} \\
\hline Estadio IVB & $\begin{array}{l}\text { Cualquier } \mathrm{T}, \mathrm{N} 3, \mathrm{M} 0 \\
\mathrm{~T} 4 \mathrm{~b} \text {, cualquier } \mathrm{N}, \mathrm{M} 0\end{array}$ & & \\
\hline Estadio IVC & $\begin{array}{c}\text { Cualquier T, cualquier } \mathrm{N}, \\
\text { M1 }\end{array}$ & & \\
\hline
\end{tabular}

\section{Cánceres de cabeza y cuello no orofaríngeos}

\section{a) Carcinoma escamoso cutáneo}

Asociado a exposición prolongada a luz ultravioleta. Un metaanálisis demuestra una mayor prevalencia de VPH en carcinoma escamoso cutáneo, sobre todo en pacientes inmunocomprometidos, sin embargo, destaca que esta asociación es no causal ${ }^{44}$. Otro metaanálisis demuestra la presencia de VPH en carcinoma escamoso cutáneo en pacientes inmunocompetentes, sobre todo en los tipos $5,8,15,17,20,24,36$ y $38^{45}$. Es importante recalcar que la presencia de VPH en tejidos tumorales no implica causalidad y pueden corresponder a infecciones transitorias, sin una relevancia biológica.

\section{b) Carcinoma escamoso rinosinusal}

El carcinoma escamoso da cuenta del $60 \%$ a $75 \%$ de las neoplasias malignas rinosinusales. Se estima que entre el $20 \%$ y el $62 \%$ se asocian a VPH, sin embargo, en estos tumores no existe metódicamente detección de VPH, por lo que la literatura es limitada. En un estudio retrospectivo de 6.458 pacientes, solo el $23 \%$ de ellos fueron analizados para VPH, siendo 446 casos $(31,5 \%)$ positivos, el genotipo más frecuente fue VPH-16 con un $77 \%$, seguido de VPH-16 junto con VPH-18 en un 9,8\%. El grupo de pacientes se caracterizó por ser más joven, de histología no queratinizada y con mayor proporción de carcinoma de alto grado ${ }^{46}$. En un estudio de 46 pacientes, el 61\% de ellos fue p16 positivo y diez de 22 muestras (45\%) VPH positivas, la sensibilidad y especificidad de p16 para la presencia de VPH fue de $90 \%$ y $67 \%$ respectivamente, en ambos casos el grupo etario fue más joven, con menor frecuencia de tabaquismo y tuvo una menor recurrencia ${ }^{47}$. Otro estudio con resultados similares destaca que el $40 \%$ de los tumores p16 positivos no tenía presencia de $\mathrm{VPH}$, por lo que esta inmunohistoquímica no sería un adecuado marcador del virus ${ }^{48}$. Notablemente, en todos los estudios citados el estatus VPH se asoció en forma significativa a un mejor pronóstico de supervivencia $(74,6 \%$ para VPH positivos vs $62,3 \%$ en VPH negativos a los 3 años, para datos pareados), por lo que los autores proponen realizar estudio VPH de forma rutinaria en estos pacientes.

\section{c) Carcinoma escamoso de la cavidad oral}

Como se describió previamente, la prevalencia del VPH en mucosa sana es alrededor del 5\% al 10\%, siendo más frecuente en hom- 
bres, y asociado a conductas sexuales de alto riesgo incluyendo el contacto oral-genital. La inflamación crónica, como la formación de sacos periodontales, favorecen la infección latente por $\mathrm{VPH}^{49}$. En lesiones premalignas, en general, la prevalencia del virus es del $22,5 \%$ (leucoplasia 20,2\%; liquen plano 23\%, fibrosis submucosa $28,6 \%$, leucoplaquia verrucosa proliferativa $24,7 \%)^{50}$. La prevalencia del VPH en carcinoma escamoso de la cavidad oral es del $23,5 \%{ }^{25}$, VPH-16 es el más frecuentemente encontrado, seguido del VPH-18 y con menor frecuencia VPH-31 y VPH-33. Los subsitios más infectados por el virus son el piso de la boca $(9 \%-42 \%)$ y la lengua $(8 \%-25 \%)^{49}$. Hasta el momento no se ha podido establecer un rol biológico relevante del virus en el proceso de carcinogénesis. En carcinomas de la cavidad oral VPH positivos, la expresión de las oncoproteínas E6/E7 es de sólo el 6\% $\%^{51}$, y la mayoría de ellos son negativos para la sobreexpresión de p16, además, p16 puede encontrarse en tumores negativos para el virus ${ }^{52}$.

\section{d) Carcinoma laríngeo}

En leucoplaquias difusas cordales existe una baja prevalencia de VPH, sin desempeñar un rol en la progresión tumoral ${ }^{53}$. En un estudio chileno de 90 pacientes, el 24 (27\%) de ellos fueron positivos para VPH, 7 de ellos del VPH-18, seguido por VPH-16 en 5 casos y VPH-52 en dos casos ${ }^{54}$. Sin embargo, a pesar de esta asociación, al igual que en cavidad oral no se ha demostrado una asociación causal. Un metaanálisis $(\mathrm{n}=2.739)^{55}$ y un reporte de serie de casos $(n=102)^{56}$ describen una actividad biológica del VPH en un 8,6\% y 5\% respectivamente, afectando a pacientes más jóvenes y con menor consumo de tabaco que aquellos VPH negativos. La expresión de p16 como marcador del virus es dudoso, ya que un bajo porcentaje de ellas son VPH positivas y, además, no se asocia a factor pronóstico, por lo que no estaría indicado su detección en biopsias laríngeas ${ }^{57}$.

\section{Prevención}

\section{Exposición laboral}

VPH-6 y VPH-11 pueden ser detectados en instrumentos médicos y en humo emana- do de cirugías. Si bien, este riesgo podría ser mayor en cirugía transoral robótica en cáncer orofaríngeo por utilizar cauterización de alta potencia, en un estudio reciente no se detectó ADN de VPH-16 en vapores de electrocauterización, máscaras quirúrgicas, aparato de succión ni brazo del robot, por lo que existiría un mínimo riesgo de exposición ocupacional a VPH en esta cirugía ${ }^{58}$.

\section{Vacunación}

Las primeras vacunas fueron diseñadas para los genotipos 16 y 18 , causantes del $70 \%$ del carcinoma cervicouterino, posteriormente se diseñaron vacunas que incluían los tipos 6 y 11 que daban cuenta del 90\% de las lesiones genitales. Una revisión Cochrane sobre la vacunación preventiva contra cáncer cervical y sus precursores indica que ambas vacunas han resultado ser seguras y con una respuesta inmunogénica potente, disminuyendo considerablemente la patología causada por los virus contenidos en la vacuna ${ }^{59}$. Sin embargo, la revisión ha sido criticada por estar incompleta, poseer sesgo de notificación e incluir ensayos sesgados, careciendo de evidencia confiable ${ }^{60}$, por lo tanto, es necesario ser cuidadoso con el alcance de los beneficios de la vacuna y extrapolación de datos. Como se mencionó previamente la vacuna cuadrivalente podría tener beneficios en pacientes con papilomatosis laríngea al disminuir la recurrencia y el intervalo quirúrgico en pacientes con enfermedad clínica activa ${ }^{17}$, así como también una disminución de la incidencia de $\mathrm{PRR}^{18}$. Un estudio realizado en jóvenes estadounidenses demostró que la vacuna se asocia a una disminución del 17\% de la carga de infecciones orales por los tipos $6,11,16$ y 18 , con una presencia de un $25 \%$ en mujeres y $6,9 \%$ en hombres. Esta modesta disminución es atribuida a la baja absorción de la vacuna ${ }^{61}$. A pesar de que estas vacunas cubren los principales genotipos involucrados en el cáncer de orofaringe, aún no existen estudios que determinen su efecto. En Chile, la vacunación cuadrivalente contra VPH se inició en el año 2014 para niñas de 9 años en esquema de 2 dosis separadas por 12 meses, y se implementó en 2019 para niños desde la misma edad ${ }^{62}$, por lo que sus efectos a nivel nacional serán vistos a largo plazo. 


\section{Discusión}

El VPH posee una alta prevalencia en distintas lesiones de cabeza y cuello, con una frecuencia variable dependiendo de la población y el subsitio analizados, siendo distintos los genotipos implicados de acuerdo con la naturaleza de la lesión. Hasta el momento solo existe un estudio chileno que determinó la prevalencia y los genotipos implicados en cáncer de laringe ${ }^{54}$, por lo que sería adecuado obtener más datos nacionales para evaluar el impacto del virus a nivel local. Si bien, el virus está presente en muchas lesiones, una relación clara de causalidad se ha establecido solo en lesiones de la piel, papilomatosis laríngea y en carcinoma orofaríngeo. Su presencia en otras lesiones tumorales podría indicar una infección transitoria, y hasta ahora no existen estudios que determinen la relevancia biológica del virus de forma aislada, como coadyuvante a otros factores de riesgo o su implicancia en el pronóstico.

Durante las últimas décadas ha existido un sostenido aumento de los carcinomas orofaríngeos relacionados a VPH, esta población se caracteriza por ser de un grupo etario más joven y sin los factores de riesgo clásicos como tabaquismo y consumo de alcohol ${ }^{32,33}$. Este carcinoma se ha constituido como una entidad propia, con una etapificación distinta debido a su buen pronóstico, por lo que actualmente existe un gran esfuerzo en el ámbito de investigación sobre protocolos de de-escalamiento ${ }^{43}$, sin embargo, hacen falta más estudios clínicos aleatorizados para aprobarlos y llevarlos a la práctica, teniendo un futuro promisorio cercano. Si bien, por su costo-efectividad y facilidad del método, la inmunohistoquímica para la proteína p16 es el método de detección más frecuentemente utilizado, es un marcador sustituto de VPH adecuado en carcinoma orofaríngeo para determinar pronóstico y no en otros tumores de cabeza y cuello. Para determinar actividad biológica del virus y no la mera infección es necesario utilizar metodologías más complejas como medición de transcritos de RNA para E6/E7 o hibridación in situ ${ }^{29}$.

Existe literatura que indica que la vacunación contra VPH podría tener utilidad en la prevención y manejo de papilomatosis laríngea con enfermedad clínicamente activa ${ }^{17,18}$, sin embargo, aún no existen reportes que evalúen su impacto en carcinoma orofaríngeo. En Chile, la vacunación es reciente sobretodo en hombres, por lo que hacen falta más estudios para determinar su efectividad en la prevención del carcinoma orofaríngeo a largo plazo.

\section{Conclusión}

El VPH está asociado a una amplia gama de lesiones tumorales benignas y malignas de cabeza y cuello, sin embargo, se ha podido determinar causalidad solo en algunas de ellas. Es necesario conocer el rol del VPH en nuestra especialidad, ya que su determinación puede tener implicancias en cuanto al manejo y pronóstico de las patologías implicadas, particularmente en carcinoma orofaríngeo. La reciente incorporación de la vacuna contra $\mathrm{VPH}$ en el programa nacional de inmunización para mujeres y hombres podría generar cambios epidemiológicos importantes, sin embargo, sus efectos podrán ser evaluados en el futuro.

\section{Bibliografía}

1. Mittal S, Banks L. Molecular mechanisms underlying human papillomavirus E6 and E7 oncoprotein-induced cell transformation. Mutat Res Rev Mutat Res. 2017;772:23-35. doi: 10.1016/j. mrrev.2016.08.001.

2. Bouvard V, Baan R, Straif K, et al. A review of human carcinogens--Part B: biological agents. Lancet Oncol. 2009;10(4):321-322. doi: 10.1016/s14702045(09)70096-8.

3. Husain N, Neyaz A. Human papillomavirus associated head and neck squamous cell carcinoma: Controversies and new concepts. J Oral Biol Craniofacial Res. 2017;7(3):198-205. doi: 10.1016/j. jobcr.2017.08.003.

4. Cheng S, Schmidt-Grimminger DC, Murant T, Broker TR, Chow LT. Differentiation-dependent up-regulation of the human papillomavirus E7 gene reactivates cellular DNA replication in suprabasal differentiated keratinocytes. Genes Dev. 1995;9(19):2335-2349. doi: 10.1101/gad.9.19.2335.

5. Doorbar J, Quint W, Banks L, et al. The biology and life-cycle of human papillomaviruses. Vaccine. 2012;30(SUPPL.5). doi: 10.1016/j. vaccine.2012.06.083.

6. Grce M, Mravak-Stipeti M. Human papillomavirusassociated diseases. Clin Dermatol. 2014;32(2):253- 
258. doi: 10.1016/j.clindermatol.2013.10.006.

7. Méndez-Martínez R, Maldonado-Frías S, VázquezVega $S$, et al. High prevalent human papillomavirus infections of the oral cavity of asymptomatic HIV-positive men. BMC Infect Dis. 2020;20(1):27. doi: 10.1186/s12879-019-4677-9.

8. Scasso F, Ferrari G, de Vincentiis GC, et al. Emerging and re-emerging infectious disease in otorhinolaryngology [Patologia infettiva emergente e riemergente in otorinolaringoiatria]. Acta Otorhinolaryngol Ital. 2018;38(2):S1-S106. doi: 10.14639/0392-100X-suppl.1-38-2018.

9. Said AK, Leao JC, Fedele S, Porter SR. Focal epithelial hyperplasia - an update. J Oral Pathol Med. 2013;42(6):435-442. doi: 10.1111/jop.12009.

10. Derkay CS, Bluher AE. Update on Recurrent Respiratory Papillomatosis. Otolaryngol Clin North Am. 2019;52(4):669-679. doi: 10.1016/j. otc.2019.03.011.

11. Fortes HR, von Ranke FM, Escuissato DL, et al. Recurrent respiratory papillomatosis: A state-ofthe-art review. Respir Med. 2017;126:116-121. doi: 10.1016/j.rmed.2017.03.030.

12. Go C, Schwartz MR, Donovan DT. Molecular transformation of recurrent respiratory papillomatosis: Viral typing and p53 overexpression. Ann Otol Rhinol Laryngol. 2003;112(4):298-302. doi: 10.1177/000348940311200402.

13. Larson DA, Derkay CS. Epidemiology of recurrent respiratory papillomatosis. APMIS. 2010;118(67):450-454. doi: 10.1111/j.1600-0463.2010.02619.x.

14. Tjon Pian Gi REA, San Giorgi MRM, SlagterMenkema L, et al. Clinical course of recurrent respiratory papillomatosis: Comparison between aggressiveness of human papillomavirus- 6 and human papillomavirus-11. Head Neck. 2015;37(11):1625-1632. doi: 10.1002/hed.23808.

15. Can NT, Tretiakova MS, Taxy JB. Natural history and malignant transformation in recurrent respiratory papillomatosis: Human papillomavirus (HPV), Dysplasia and an autopsy review. Fetal Pediatr Pathol. 2015;34(2):80-90. doi: 10.3109/15513815.2014.968271.

16. Donne AJ, Hampson L, Homer JJ, Hampson IN. The role of HPV type in Recurrent Respiratory Papillomatosis. Int J Pediatr Otorhinolaryngol. 2010;74(1):7-14. doi: 10.1016/j.ijporl.2009.09.004.

17. Novakovic D, Cheng ATL, Zurynski Y, et al. A Prospective Study of the Incidence of JuvenileOnset Recurrent Respiratory Papillomatosis after Implementation of a National HPV Vaccination Program. J Infect Dis. 2018;217(2):208-212. doi: 10.1093/infdis/jix498.

18. Dion GR, Teng S, Boyd LR, et al. Adjuvant Human Papillomavirus Vaccination for Secondary Prevention: A Systematic Review. JAMA Otolaryngol Head Neck Surg. 2017;143(6):614-622. doi: 10.1001/ jamaoto.2016.4736.

19. Mohajeri S, Lai C, Purgina B, et al. Human papillomavirus: An unlikely etiologic factor in sinonasal inverted papilloma. Laryngoscope. 2018;128(11):2443-2447. doi: 10.1002/lary.27207.

20. Stoddard DG Jr, Keeney MG, Gao G, Smith DI, García JJ, O’Brien EK. Transcriptional activity of HPV in inverted papilloma demonstrated by in situ hybridization for E6/E7 mRNA. Otolaryngol Head Neck Surg. 2015;152(4):752-758. doi: 10.1177/0194599815571285.

21. Scheel A, Lin GC, Mchugh JB, et al. Human papillomavirus infection and biomarkers in sinonasal inverted papillomas: Clinical significance and molecular mechanisms. Int Forum Allergy Rhinol. 2015;5(8):701-707. doi: 10.1002/alr.21524.

22. Zhao RW, Guo ZQ, Zhang RX. Human papillomavirus infection and the malignant transformation of sinonasal inverted papilloma: A meta-analysis. J Clin Virol. 2016;79:36-43. doi: 10.1016/j.jcv.2016.04.001.

23. Wang H, Zhai C, Liu J, et al. Low prevalence of human papillomavirus infection in sinonasal inverted papilloma and oncocytic papilloma. Virchows Arch. 2020;476(4):577-583. doi: 10.1007/s00428-01902717-3.

24. Fulla M, Szafarowski T, Frias-Gomez J, et al. Human Papillomavirus and Factors Associated with Recurrence in Sinonasal Inverted Papillomas from Poland and Spain. Head Neck Pathol. 2020. doi: 10.1007/s12105-019-01125-y.

25. Kreimer AR, Clifford GM, Boyle P, Franceschi S. Human Papillomavirus Types in Head and Neck Squamous Cell Carcinomas Worldwide: A Systematic Review. Cancer Epidemiol Biomarkers Prev. 2005;14(2):467-475. doi: 10.1158/1055-9965.EPI-040551.

26. Wagner S, Sharma SJ, Wuerdemann N, et al. Human Papillomavirus-Related Head and Neck Cancer. Oncol Res Treat. 2017;40(6):334-340. doi: $10.1159 / 000477252$.

27. de Martel C, Plummer M, Vignat J, Franceschi S. Worldwide burden of cancer attributable to HPV by site, country and HPV type. Int J Cancer. 2017;141(4):664-670. doi: 10.1002/ijc.30716.

28. Prigge ES, Arbyn M, von Knebel Doeberitz M, Reuschenbach M. Diagnostic accuracy of p16INK4a immunohistochemistry in oropharyngeal squamous cell carcinomas: A systematic review and metaanalysis. Int J Cancer. 2017;140(5):1186-1198. doi: 10.1002/ijc.30516.

29. Paver EC, Currie AM, Gupta R, Dahlstrom JE. Human papilloma virus related squamous cell carcinomas of the head and neck: diagnosis, clinical implications and detection of HPV. Pathology. 2020;52(2):179-191. doi: 10.1016/j. pathol.2019.10.008. 
30. Mallen-St Clair J, Alani M, Wang MB, Srivatsan ES. Human papillomavirus in oropharyngeal cancer: The changing face of a disease. Biochim Biophys Acta. 2016;1866(2):141-150. doi: 10.1016/j. bbcan.2016.07.005.

31. Sano D, Oridate N. The molecular mechanism of human papillomavirus-induced carcinogenesis in head and neck squamous cell carcinoma. Int J Clin Oncol. 2016;21(5):819-826. doi: 10.1007/s10147-0161005-x.

32. Castellsagué X, Alemany L, Quer M, et al. HPV Involvement in Head and Neck Cancers: Comprehensive Assessment of Biomarkers in 3680 Patients. J Natl Cancer Inst. 2016;108(6):djv403. doi: 10.1093/jnci/djv403.

33. Gillison ML, Chaturvedi AK, Anderson WF, Fakhry C. Epidemiology of Human Papillomavirus-Positive Head and Neck Squamous Cell Carcinoma. $J$ Clin Oncol. 2015;33(29):3235-3242. doi: 10.1200/ JCO.2015.61.6995.

34. Pytynia KB, Dahlstrom KR, Sturgis EM. Epidemiology of HPV-associated oropharyngeal cancer. Oral Oncol. 2014;50(5):380-386. doi: 10.1016/j.oraloncology.2013.12.019.

35. Cook EE, Gershman ST, Kim JJ, Tamimi RM, Klevens RM, Holmes MD. Trends of two HPVassociated cancers in Massachusetts: cervical and oropharyngeal cancer. Cancer Causes Control. 2018;29(4-5):435-443. doi: 10.1007/s10552-018-10161.

36. Marur S, Souza GD', Westra WH, Forastiere AA, Professor A, Hopkins J. HPV-associated Head and Neck Cancer: A Virus-related Cancer Epidemic - A Review of Epidemiology, Biology, Virus Detection and Issues in Management. Lancet Oncol. 2010;11(8):781-789. doi: 10.1016/S14702045(10)70017-6.

37. Nci, Seer. Head and Neck: Summary Stage 2018 Coding Manual v2.0.

38. Tham T, Ahn S, Frank D, Kraus D, Costantino P. Anatomical subsite modifies survival in oropharyngeal squamous cell carcinoma: National Cancer Database study. Head Neck. 2020;42(3):434445. doi: 10.1002/hed.26019.

39. Ang KK, Harris J, Wheeler R, et al. Human papillomavirus and survival of patients with oropharyngeal cancer. N Engl J Med. 2010;363(1):2435. doi: 10.1056/NEJMoa0912217.

40. O'Sullivan B, Huang SH, Su J, et al. Development and validation of a staging system for HPVrelated oropharyngeal cancer by the International Collaboration on Oropharyngeal cancer Network for Staging (ICON-S): a multicentre cohort study. Lancet Oncol. 2016;17(4):440-451. doi: 10.1016/S14702045(15)00560-4.

41. Huang SH, O'Sullivan B. Overview of the 8th Edition TNM Classification for Head and Neck Cancer. Curr
Treat Options Oncol. 2017;18(7):40. doi: 10.1007/ s11864-017-0484-y.

42. Sandulache VC, Wilde DC, Sturgis EM, Chiao EY, Sikora AG. A Hidden Epidemic of "Intermediate Risk" Oropharynx Cancer. Laryngoscope Investig Otolaryngol. 2019;4(6):617-623. doi: 10.1002/lio2.316.

43. Geiger JL, Ku JA. Postoperative Treatment of Oropharyngeal Cancer in the Era of Human Papillomavirus. Curr Treat Options Oncol. 2019;20(3):20. doi: 10.1007/s11864-019-0620-y.

44. Wang J, Aldabagh B, Yu J, Arron ST. Role of human papillomavirus in cutaneous squamous cell carcinoma: a meta-analysis. J Am Acad Dermatol. 2014;70(4):621-629. doi: 10.1016/j.jaad.2014.01.857.

45. Chahoud J, Semaan A, Chen Y, et al. Association between -genus human papillomavirus and cutaneous squamous cell carcinoma in immunocompetent individuals-a meta-analysis. JAMA Dermatology. 2016;152(12):1354-1364. doi: 10.1001/jamadermatol.2015.4530.

46. Oliver JR, Lieberman SM, Tam MM, et al. Human papillomavirus and survival of patients with sinonasal squamous cell carcinoma. Cancer. 2020;126(7):1413-1423. doi: 10.1002/cncr.32679.

47. Cohen E, Coviello C, Menaker S, et al. P16 and human papillomavirus in sinonasal squamous cell carcinoma. Head Neck. 2020. doi: 10.1002/ hed.26134.

48. Jiromaru R, Yamamoto H, Yasumatsu R, et al. HPVrelated Sinonasal Carcinoma: Clinicopathologic Features, Diagnostic Utility of p16 and Rb Immunohistochemistry, and EGFR Copy Number Alteration. Am J Surg Pathol. 2020;44(3):305-315. doi: 10.1097/PAS.0000000000001410.

49. Hübbers CU, Akgül B. HPV and cancer of the oral cavity. Virulence. 2015;6(3):244-248. doi: 10.1080/21505594.2014.999570.

50. de la Cour CD, Sperling CD, Belmonte F, Syrjänen S, Kjaer SK. Human papillomavirus prevalence in oral potentially malignant disorders: Systematic review and meta-analysis. Oral Dis. 2020. doi: 10.1111/ odi.13322.

51. Lingen MW, Xiao W, Schmitt A, et al. Low etiologic fraction for high-risk human papillomavirus in oral cavity squamous cell carcinomas. Oral Oncol. 2013;49(1):1-8. doi: 10.1016/j. oraloncology.2012.07.002.

52. Reuschenbach M, Kansy K, Garbe K, et al. Lack of evidence of human papillomavirus-induced squamous cell carcinomas of the oral cavity in southern Germany. Oral Oncol. 2013;49(9):937-942. doi: 10.1016/j.oraloncology.2013.03.451.

53. Waters HH, Seth R, Hoschar AP, Benninger MS. Does HPV have a presence in diffuse high grade pre-malignant lesions of the larynx? Laryngoscope. 2010;120 Suppl 4:S201. doi: 10.1002/lary.21668.

54. Torrente M, Yeomans L, Abud M, Ojeda JM. 
Distribución relativa de genotipos de virus papiloma humano en muestras de carcinoma escamoso de laringe. Rev Otorrinolaringol Cir Cabeza Cuello. 2009;69(2):87-92. doi: 10.4067/s071848162009000200002 .

55. Ndiaye C, Mena M, Alemany L, et al. HPV DNA, E6/E7 mRNA, and p16INK4a detection in head and neck cancers: a systematic review and metaanalysis.[Erratum appears in Lancet Oncol. 2015 Jun;16(6):e262]. Lancet Oncol. 2014;15(12):13191331. doi: https://dx.doi.org/10.1016/S14702045(14)70471-1.

56. Halec G, Holzinger D, Schmitt M, et al. Biological evidence for a causal role of HPV16 in a small fraction of laryngeal squamous cell carcinoma. Br J Cancer. 2013;109(1):172-183. doi: 10.1038/ bjc.2013.296.

57. Wittekindt C, Wuerdemann N, Gattenlöhner S, et al. The role of high-risk human papillomavirus infections in laryngeal squamous cell carcinoma. Eur Arch Oto-Rhino-Laryngology. 2017;274(11):38373842. doi: 10.1007/s00405-017-4718-1.

58. Subbarayan RS, Shew M, Enders J, Bur AM, Thomas SM. Occupational exposure of oropharyngeal human papillomavirus amongst otolaryngologists. Laryngoscope. 2019. doi: 10.1002/lary.28383.

59. Arbyn M, Xu L, Simoens C, Martin-Hirsch PPL. Prophylactic vaccination against human papillomaviruses to prevent cervical cancer and its precursors. Cochrane Database Syst Rev. 2018;2018(5). doi: 10.1002/14651858.CD009069.pub3.

60. Jørgensen L, Gøtzsche PC, Jefferson T. The Cochrane HPV vaccine review was incomplete and ignored important evidence of bias. BMJ EvidenceBased Med. 2018;23(5):165-168. doi: 10.1136/ bmjebm-2018-111012.

61. Chaturvedi AK, Graubard BI, Broutian T, et al. Effect of prophylactic human papillomavirus (HPV) vaccination on oral HPV infections among young adults in the United States. J Clin Oncol. 2018;36(3):262-267. doi: 10.1200/JCO.2017.75.0141.

62. Autoridades de Salud inician primera vacunación en niños para prevenir el Virus del Papiloma Humano (VPH) - Ministerio de Salud - Gobierno de Chile. https://www.minsal.cl/autoridades-de-salud-inicianprimera-vacunacion-en-ninos-para-prevenir-elvirus-del-papiloma-humano-vph/. Accessed April 27, 2020. 